\title{
Far-UV emissions from the SL9 impacts with Jupiter
}

\author{
G.E. Ballester ${ }^{1}$, W.M. Harris ${ }^{1}$, G.R. Gladstone ${ }^{2}$, J.T. Clarke ${ }^{1}$, R. Prangé $\dot{e}^{3,4}$, \\ P.D. Feldman ${ }^{5}$, M.R. Combi ${ }^{1}$, C. Emerich ${ }^{3}$, D.F. Strobel ${ }^{5}$, A. Talavera ${ }^{6}$, S.A. \\ Budzien $^{7}$, M.B. Vincent ${ }^{1}$, T.A. Livengood ${ }^{8}$, K.L. Jessup ${ }^{1}$, M.A. McGrath ${ }^{9}$, \\ D.T. Hall ${ }^{5}$, J.M. Ajello ${ }^{10}$, L. ben Jaffel ${ }^{4}$, D. Rego ${ }^{1}$, G. Fireman ${ }^{11}$, L. \\ Woodney $^{12}$, S. Miller ${ }^{13}$, and X. Liu ${ }^{9}$
}

\begin{abstract}
Observations with the International Ultraviolet Explorer (IUE) during the impacts of the fragments of comet $D /$ Shoemaker-Levy 9 with Jupiter show far-UV emissions from the impact sites within a $\sim 10$ min time scale. Positive detections of $\mathrm{H}_{2}$ Lyman and Werner band (1230-1620 $\AA)$ and H-Ly $\alpha$ emissions are made for impacts $\mathrm{K}$ and $\mathrm{S}$, and marginally for $\mathrm{P}$. No thermal continuum is observed. The radiated farUV output was $>10^{21}$ ergs. The $\mathrm{H}_{2}$ spectrum is consistent with electron collisional excitation if significant $\mathrm{CH}_{4}$ absorption is included. Such emissions could result from plasma processes generated by the impacts. Non-thermal excitation by the high altitude entry and explosion shocks may also be relevant. Emissions by $\mathrm{Al}^{+}(1671 \AA)$ and $\mathrm{C}(1657 \AA)$ of cometary origin are tentatively identified.
\end{abstract}

\section{Introduction}

During the July 1994 comet collision with Jupiter large amounts of mass and energy were deposited in the upper atmosphere. This region is easily probed in the far-UV $(\sim 1200-2000 \AA)$ because Rayleigh scattering and hydrocarbon absorption isolate it from lower altitudes, and its main $\mathrm{H}$ and $\mathrm{H}_{2}$ components have strong signatures at these wavelengths that have been extensively used for the past 16 years to study the auroral processes that connect the upper atmosphere with the magnetosphere. Auroral-like emissions from fieldaligned currents connecting cometary dust to the ionosphere during the comet passage through the magneto-

\footnotetext{
' Space Physics Research Lab., University of Michigan, Ann Arbor

${ }^{2}$ Southwest Research Institute, San Antonio, TX

${ }^{3}$ Institute d'Astrophysique Spatiale, Orsay, France

${ }^{4}$ Institute d'Astrophysique de Paris, Paris, France

${ }^{5}$ Johns Hopkins University, Baltimore, MD

${ }^{6}$ IUE Observatory, Villafranca del Castillo, Madrid Spain

${ }^{7}$ Naval Research Lab., Washington, DC

${ }^{8}$ Goddard Space Flight Center, Greenbelt, MD

${ }^{9}$ Space Telescope Science Institute, Baltimore, MD

${ }^{10}$ Jet Propulsion Lab., Pasadena, CA

${ }^{11}$ Computer Sciences Corporation, Lanham, MD

${ }^{12}$ University of Maryland, College Park, MD

${ }^{13}$ University College London, London, UK
}

\section{Copyright 1995 by the American Geophysical Union.}

sphere may have been observed with the Hubble Space Telescope (HST) [Clarke et al., 1995; Prangé et al., 1995, Ip and Prangé, 1994].

We report in this letter spectroscopic observations with the IUE satellite made during the impacts of fragments $\mathrm{K}, \mathrm{S}$ and $\mathrm{P} 2$ that show far-UV emissions by $\mathrm{H}$ and $\mathrm{H}_{2}$ associated with the impacts themselves. Tentative detections of $\mathrm{C}$ and $\mathrm{Al}^{+}$emissions are also presented. We analyze the emissions within the context of possible excitation processes. These data are part of the extensive set of IUE observations of the comet collision.

\section{Observational results}

Impacts $\mathrm{K}, \mathrm{P} 2$ and $\mathrm{S}$ were observed for $\sim 20 \mathrm{~min}$ centered around impact time (Table 1). The IUE SWP camera functioned as an array spectrograph, with the long axis of the aperture nearly perpendicular to the spectral dispersion direction providing spatial discrimination. The aperture was centered at latitude $-45^{\circ}$ on the approaching limb, with the spatial lines of spectra fairly tangent to the limb (Fig. 1). With the $\sim 1^{\prime \prime}$ pointing accuracy and the telescope's $\sim 3^{\prime \prime}$ PSF, contamination by southern auroral emission was unlikely (Fig. 1). Planet background signal from scattered sunlight and weak dayglow hydrogen emissions [McGrath et al., 1989] was relatively low due to limb darkening.

The impact emissions were extracted with a method for subtracting the planet background from IUE Jovian auroral data based on the spatial information and shown in Fig. 1 [Harris et al., 1995]. Representative limb spectra were also obtained with a less accurate method described in Fig. 2.

The highest signal to noise ratio $(\mathrm{s} / \mathrm{n})$ was obtained for impact $K$. Combining the spectrum and the spatial extractions we identified emissions by $\mathrm{H}_{2}(\sim 1230$ $1620 \AA)$ and $\mathrm{H}-\mathrm{Ly} \alpha(1216 \AA)$. The $\mathrm{H}_{2}$ signal was at least a $3 \sigma$ detection near $1600 \AA$, but decreased to $\sim 1 \sigma$ at shorter wavelengths. The $s / n$ for impact $S$ was somewhat lower, and the smallest fragment P2 impact showed only a hint of emission around $1600 \AA$ and Ly $\alpha$. No emissions were detected in the background exposure. $\mathrm{A} \sim 4 \sigma$ feature at $1671 \AA$ appeared at the limb for impact $K$, but was not above the noise for impact $\mathrm{S}$. We identified this emission as an $\mathrm{Al}^{+}$resonance line. The spatial extractions also showed distinct C $1657 \AA$ emissions at the limb for both $\mathrm{K}$ and $\mathrm{S}$, with $\mathrm{a} \sim 2.3 \sigma$ detection for impact $\mathrm{K}$ (Fig. 1) though only $\sim 1.4 \sigma$ for $\mathrm{S}$. (The $\mathrm{C}$ feature for $\mathrm{K}$ appears as only $\mathrm{a} \sim 1 \sigma$ detec- 
Table 1. Exposure Times and Lower Limit Energies

\begin{tabular}{cccccccccc}
\hline Fragment & $\begin{array}{c}\text { Expo. Start } \\
\text { (UT) }\end{array}$ & $\begin{array}{c}\text { Length } \\
(\mathrm{min})\end{array}$ & $\begin{array}{c}\text { Impact } \\
\text { time }^{a}\end{array}$ & $\begin{array}{c}\text { Post-I } \\
(\mathrm{min})\end{array}$ & $\mathrm{H}_{2}$-Ly & $\mathrm{H}_{2}$-W & $\begin{array}{c}\text { Energy (ergs) } \\
\text { H-Ly } \alpha\end{array}$ & $\mathrm{C}$ & $\mathrm{Al}^{+}$ \\
\hline K & 19 Jul 10:12:54 & 23 & $10: 24$ & 12 & $3 \times 10^{20}$ & $1 \times 10^{20}$ & $4 \times 10^{20}$ & $4 \times 10^{19}$ & $6 \times 10^{19}$ \\
P2 & 20 Jul 15:16:08 & 20 & $15: 23$ & 13 & $6 \times 10^{19}$ & - & $1 \times 10^{20}$ & - & - \\
Background & 21 Jul 14:09:46 & 20 & - & - & - & - & - & - & - \\
S & 21 Jul 15:01:56 & 20 & $15: 15$ & 7 & $4 \times 10^{20}$ & $1 \times 10^{20}$ & $3 \times 10^{20}$ & $2 \times 10^{19}$ & $2 \times 10^{10}$ \\
\hline
\end{tabular}

"From D. Yeomans and P. Chodas, JPL Report, Pasadena, CA, 13 Oct 1994.

' $\mathrm{H}_{2}-\mathrm{Ly}(1550-1620 \AA) ; \mathrm{H}_{2}-\mathrm{W}(1230-1300 \AA) ; \mathrm{H}-\mathrm{Ly} \alpha(1200-1230 \AA) ; \mathrm{C}(1652-1662 \AA) ; \mathrm{I}^{+}(1666-1676 \AA)$.

tion in the less accurate spectrum in Fig. 2.) These $\mathrm{C}$ and $\mathrm{Al}^{+}$emissions are tentative detections. Their uncertainties were estimated by extracting the same features in the P2 and background exposures and taking $1 \sigma$ to be the standard deviation from the mean; this included any fixed-pattern detector noise. Other emissions, such as from $\mathrm{CO}, \mathrm{O}$ or $\mathrm{S}$, are not apparent above the noise level. No continuum flux was detected, and a 0.002 photons $\mathrm{cm}^{-2} \mathrm{sec}^{-1} \AA^{-1}$ upper limit is set at $1700 \AA$ for post-impact $\mathrm{K}$.

There are two major uncertainties in deriving emission intensities: the location and extent of the source with respect to the limb and its duration. Net radiated far-UV energies detected during the exposures are listed in Table 1. These represent lower limits but are already quite large, about $10^{21}$ ergs total (though only $10^{-7}$ of a $1 \mathrm{~km}, 1 \mathrm{gm} \mathrm{cm}^{-3}$ impactor's energy). Table 2 lists lower limits for the integrated fluxes evaluated using the post-impact exposure times. Determination of the source area is limited by the combined $\sim 5^{\prime \prime}$ PSF of the telescope and SWP camera. The derived spatial FWHM of the emission features are also $\sim 4-5^{\prime \prime}$, with the $\mathrm{H}$-Ly $\alpha$ and the bright, narrow $\mathrm{H}_{2}$ feature at $1610 \AA$ appearing point-like along the dispersion. Given the low $\mathrm{s} / \mathrm{n}$, the source could thus range from point-like to not wider than $\sim 2^{\prime \prime}$ or $3^{\prime \prime}$. Lower limit surface brightnesses were thus obtained for a $2^{\prime \prime}(7330 \mathrm{~km})$ diameter source and post-impact exposure times (Table 2). The tens of kilo-Rayleighs (kR) derived for the hydrogen emissions are large, similar to average auroral values, but the uncertainties involved could easily bring the brightnesses to Mega-Rayleigh values.

\section{Excitation of the $H$ and $H_{2}$ emissions}

The $\mathrm{H}$ and $\mathrm{H}_{2}$ emissions observed by IUE may be attributable to collisional excitation by plasma processes or to non-thermal shock emission. We examine briefly these and other mechanisms.

Collisional excitation/plasma processes. The $\mathrm{H}_{2}$ spectrum resembles a spectrum collisionally excited by electrons if significant absorption by $\mathrm{CH}_{4}$, the dominant stratospheric component, is included (Fig. 3). Electrons would also produce $\mathrm{H}-\mathrm{Ly} \alpha$ emission by $\mathrm{H}_{2}$ dissociative excitation and direct $\mathrm{H}$ excitation. Various constraints can be derived for the case of collisional excitation. The observed H-Ly $\alpha / \mathrm{H}_{2}$ Lyman-band ratio and the hydrocarbon absorption are similar to those of the Jovian aurora [Harris et al., 1995; Gladstone and Skinner, 1989; Livengood et al., 1990]. The observed emissions would thus result from excitation of $\mathrm{H}$ and $\mathrm{H}_{2}$ by electrons having energies in excess of a few tens of $\mathrm{eV}$. For the $\mathrm{K}$ impact and $20 \mathrm{eV}$ electrons of $1 \mathrm{~cm}^{-3}$ density, a column of $\sim 6 \times 10^{19} \mathrm{~cm}^{-2}$ of $\mathrm{H}_{2}$ and of at least $10^{17} \mathrm{~cm}^{-2}$ of $\mathrm{CH}_{4}$ are required. The emission source may have been near the altitude of the $\mathrm{CH}_{4}$ homopause, or a higher altitude emission beyond the limb would have been subject to line of sight absorption from lower altitude $\mathrm{CH}_{4}$.

Collisional excitation is typical of plasma processes, for which some information can be derived from comparison with other observations. The surprising detection by the WFPC2 camera on HST of far-UV emission arcs on the northern hemisphere magnetically conjugate to the plume area of fragment $\mathrm{K}$, as well as around the plume itself, showed that the impacts drove plasma processes lasting for an hour time scale [Clarke et al., 1995]. Near-IR ionospheric $\mathrm{H}_{3}^{+}$emissions with similar characteristics were also imaged for impact K [McGregor, 1995]. The WFPC2 emissions decreased significantly in a $\sim 10 \mathrm{~min}$ time scale, and were $\sim 250 \mathrm{kR}$ over $\sim 2000 \times 20,000 \mathrm{~km}^{2}$, an area similar to that used for the IUE lower limits. If the IUE and WFPC2 emissions were driven by the same process, considering the expansion of the plume between the IUE and WFPC2 detections, the IUE emissions could agree with the WFPC2 decay time scale or could accommodate instead stronger emission near impact time.

To explain the WFPC2 emissions, Hill and Dessler, [1995] proposed a mechanism where the flow of the plume reentering the ionosphere and crossing magnetic field lines would create temporary field-aligned currents in the ionosphere. Potential drops associated with these currents would accelerate particles and excite the ambient atmosphere. Although $\sim 6 \mathrm{~min}$ of plume fallback (inferred to have started $\sim 6 \mathrm{~min}$ after impact from many IR observations) was observed for $\mathrm{K}$ and $\mathrm{P} 2$, only $\sim 1 \mathrm{~min}$ was observed for $\mathrm{S}$. Therefore, to produce the IUE emissions, the generation of plasma effects with sufficient energy output may also be required for an earlier impact stage. The rising explosion shock and fireball are good candidates because their large envelope expanded through the ionosphere across magnetic field lines and should have carried significant ionization.

The ionization and motion through magnetic field lines of the fragment entry should have also created ionospheric currents, but with too low an energy output to produce noticeable effects [Kellogg, 1994]. It is also unlikely that the IUE emissions were produced by perturbation of the radiation belt particles into the loss cone from plasma waves generated in the ionosphere, since more than $\sim 20 \%$ efficiency would be needed for the estimated $\sim 10^{22}$ ergs output [Hill and Dessler, 1995]. 

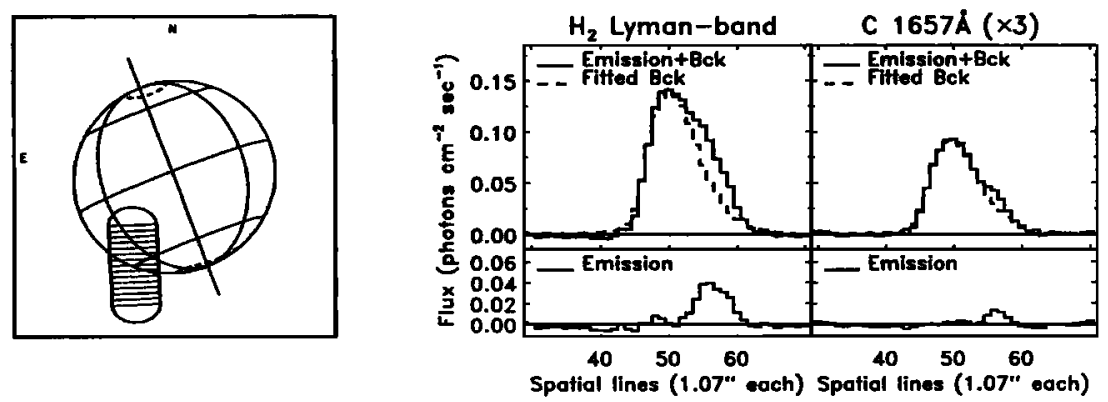

Figure 1. LEFT: Viewing geometry with the large $21^{\prime \prime} \times 9^{\prime \prime}$ IUE aperture oriented with the spatial $1.07^{\prime \prime} \times 9^{\prime \prime}$ lines of spectra $\sim 25^{\circ}$ to the tangent of Jupiter's limb at latitude $-45^{\circ}$. Latitude and longitude lines are drawn every $45^{\circ}$. Auroral ovals projecting to 30 Jovian radii $\left[\mathrm{O}_{6}\right.$ magnetic field, Connerney, p.c.] plotted in dashed lines for the $\mathrm{K}$ and $\mathrm{S}$ exposures are well outside the aperture. RIGHT: Spatial flux extraction of the $\mathrm{H}_{2}$ Lyman-band (left) and $\mathrm{C} 1657 \AA$ (right) emission of impact $\mathrm{K}$. Spatial profiles along the aperture's long axis are obtained by co-adding signal in the spectral direction within a bandpass. Flux beyond the aperture edges at lines $\sim 45$ and $\sim 65$ is due to the PSF. Top panels show the profiles for the emission region and the fitted planet background (dashed line; extracted from the 1700-1800 $\AA$ region devoid of limb emission and saturation and scaled and shifted to match the original profile at the shoulder opposite to the limb); bottom panels show the resulting emission profile. Earth H-Ly $\alpha$ background was subtracted by fitting a spatial profile derived from an average of geocoronal exposures. Fluxes correspond to the full exposure time.

Finally, strong bursts of X-ray emission near the northern auroral regions were detected near the times of impacts $\mathrm{K}$ and P2 but not S [Waite et al., 1995]. The relative intensities of the coincident IUE emissions on the south disagree with these, a constraint for any possible common plasma process.

Excitation from other impact processes. The non-thermal shock formed during the early fragment entry in the $\sim 1600-2000 \mathrm{~km}$ altitude region should have produced (bolide) line emission by shocked atmospheric and ablated gases [Chevalier and Sarazin, 1994]. In particular, H-Ly $\alpha$ and $\mathrm{H}_{2}$ emission are expected, although

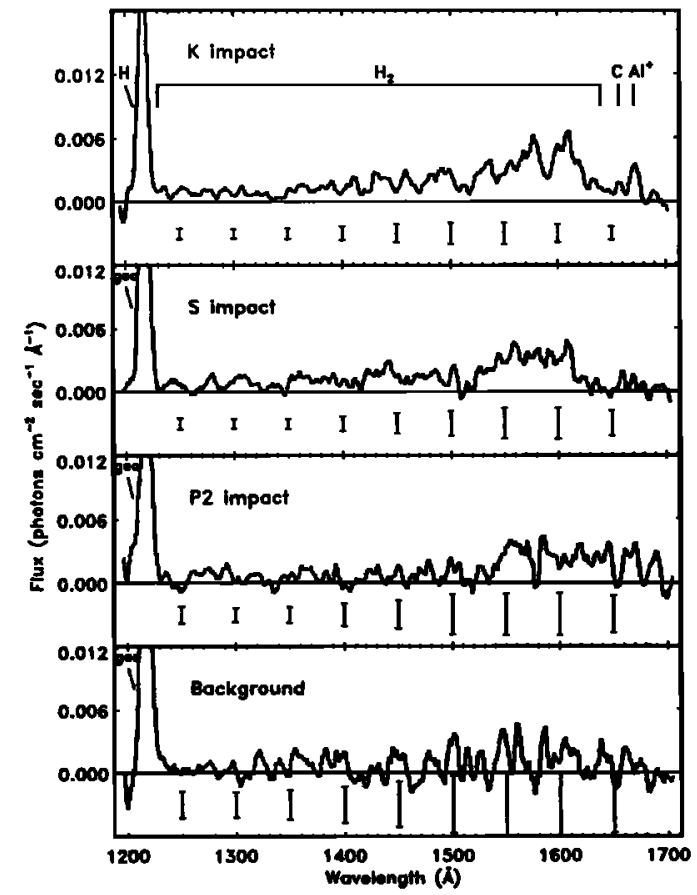

Figure 2. Spectra (smoothed by 5 points) for the K, $S$ and $\mathrm{P} 2$ impacts and background exposures (top to bottom). The $\mathrm{K}$ spectrum shows the identified emissions. The subtraction of the planet background (from a planet spectrum scaled to match the limb at $\sim 1700 \AA$ ) is somewhat inaccurate, especially above $1600 \AA$ where the reflected solar spectrum increases sharply. Geocoronal H-Ly $\alpha$ has not been subtracted, but is quite low for $\mathrm{K}$. The $\pm 1 \sigma$ error bars are estimated from the noise amplitude and camera background level. Fluxes correspond to the full exposure time.
Table 2. Lower Limit Fluxes and Brightnesses for Post-impact Exposure Times and a 2" Source Area

\begin{tabular}{cccccc}
\hline \multirow{2}{*}{ Frag. } & \multicolumn{5}{c}{ Flux (photons $\mathrm{cm}^{-2} \mathrm{sec}^{-1}$ ) / Brightness (kR) } \\
& $\mathrm{H}_{2}-\mathrm{Ly}$ & $\mathrm{H}_{2}-\mathrm{W}$ & $\mathrm{H}-\mathrm{Ly} \alpha$ & $\mathrm{C}$ & $\mathrm{Al}^{+}$ \\
\hline $\mathrm{K}$ & $\mathbf{0 . 5 2 / 8 9}$ & $\mathbf{0 . 1 7 / 2 9}$ & $\mathbf{0 . 4 5 / 7 7}$ & $\mathbf{0 . 0 5 / 9}$ & $0.08 / 14$ \\
$\mathrm{~S}$ & $\mathbf{0 . 9 9 / 1 6 8}$ & $\mathbf{0 . 2 2 / 3 7}$ & $\mathbf{0 . 4 9 / 8 3}$ & $0.05 / 8$ & $0.06 / 10$ \\
$\mathrm{P} 2$ & $0.09 / 15$ & & $0.13 / 22$ & & \\
\hline
\end{tabular}

the shape of the $\mathrm{H}_{2}$ spectrum is unknown. For impacts $\mathrm{K}$ and $\mathrm{S}$ only altitudes above $\sim 830$ and $\sim 650 \mathrm{~km}$ projected above the limb of the $\mathrm{CH}_{4}$ homopause, where it is not clear if the atmosphere would be dense enough (pressures $\lessgtr$ nanobars) to produce significant emission. The first precursors observed from Earth are potential confirmation of such bolide emissions [e.g., Hammel et al., 1995]. The outgoing shock from the energetic explosion could have also produced non-thermal emission after reaching high altitudes [e.g., Boslough et al., 1994]. Chevalier and Sarazin [1994] estimated an energy flux for the shock front at $\sim 500 \mathrm{~km}$ altitude of $10^{20}$ ergs sec${ }^{-1} \mathrm{~km}^{-2}$ which is sizable given the large shock front area, and they inferred that the phenomenon should have extended to $\sim 2000 \mathrm{~km}$ altitude.

Other possible mechanisms associated with the observed impact stages were considered and ruled out. 1) Excitation by direct comet-atmosphere collisions above $\sim 1600 \mathrm{~km}$ should have been insignificant. 2) The extremely bright UV thermal radiation from the thermalized entry shock observed with Galileo could have been resonantly and fluorescently scattered by upper atmospheric $\mathrm{H}$ and $\mathrm{H}_{2}$ (which fluoresces photons of $\lambda<1100 \AA$ ), but was produced so deep that all the light below $\sim 1450 \AA$ should have been absorbed by stratospheric $\mathrm{CH}_{4}$. 3) A process that seems unlikely but needs better assessment is excitation within the fireball by high-energy $\left(Z^{20} \mathrm{eV}\right)$ tail electrons or by unknown suprathermal electrons. Sufficiently high electron densities would be needed, and it is not clear that they could be attained in the relatively cold $(T \leqslant 2000 \mathrm{~K})$ fireballs that were visible from Earth [e.g., Nicholson et al., 1995]. 4) Finally, fluorescence of solar lines by $\mathrm{H}_{2}$ in the plumes would yield an $\mathrm{H}_{2}$ spectrum significantly 


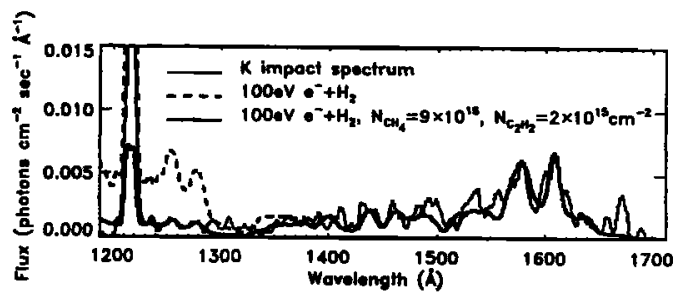

Figure 3. K-impact spectrum (thin line) versus a pure laboratory $\mathrm{H}_{2}$ spectrum excited by $100 \mathrm{eV}$ electrons (dashed line), and versus the same $\mathrm{H}_{2}$ spectrum but including absorption by $\mathrm{CH}_{4}$ and $\mathrm{C}_{2} \mathrm{H}_{2}$ to match the observation (thick line).

different than observed [e.g. Feldman and Fastie, 1973; Jordan et. al., 1978]. The model of Gladstone [1988] for radiative transfer calculations of resonant scattering of the solar H-Ly $\alpha$ line was adapted for various columns of $\mathrm{H}$ in a plume containing $\mathrm{CH}_{4}$ at the $2 \times 10^{-3}$ lower atmosphere $\mathrm{C} / \mathrm{H}$ mixing ratio $\left(60^{\circ}\right.$ zenith angle and $3.5 \times 10^{11}$ photons $\mathrm{cm}^{-2} \mathrm{sec}^{-1}$ solar Ly $\alpha$ flux at $1 \mathrm{AU}$ ). The contribution from this source reaches only $\sim 10 \mathrm{kR}$ for columns $>10^{18} \mathrm{~cm}^{-2}$.

\section{Excitation of the $\mathrm{C}$ and $\mathrm{Al}^{+}$emissions}

The stratosphere should be fairly transparent above $\sim 1450 \AA$, so the bright thermal emission from hot shocks should have reached high altitudes where it could have been resonantly scattered by $\mathrm{C}$ and $\mathrm{Al}^{+}$ablated during the comet entry or in the coma. In particular, for the hot $\sim 7800 \mathrm{~K}, 1.6 \mathrm{sec}, 40 \mathrm{~km}^{2}$ entry shock source observed for impact $\mathrm{G}$ by Galileo [Hord et al., 1995], C and $\mathrm{Al}^{+}$columns of roughly $10^{13}$ and $10^{12} \mathrm{~cm}^{-2}$ would be needed for a scattering source at a distance of $500 \mathrm{~km}$ to yield the brightnesses derived for impact $\mathrm{K}$ (with a correction of $720 / 1.6$ for the emission time). The exact values depend on the geometry and Rayleigh scattering, but these columns would have a cometary origin. The detection of $\mathrm{Al}^{+}$would not be surprising given that $\mathrm{Al}$ and $\mathrm{Mg}$ are often seen together in astrophysical sources, and $\mathrm{Mg}^{+}$emission was detected on the $\mathrm{G}$ impact site [Noll et al., 1995]. (The non-detection of C $1561 \AA$ emission would be due to wavelength dependence of the thermal flux and the absorption oscillator strength.) Another possible source of the emissions could be non-thermal excitation of ablated $\mathrm{C}$ and $\mathrm{Al}^{+}$ by the entry and outgoing shocks.

Processes that were ruled out include: 1) Those involving electron excitation (require too much cometary $\mathrm{Al}^{+}$and $\mathrm{C}$ due to large optical thickness effects). 2) Electron dissociative excitation of $\mathrm{CH}_{4}$ and $\mathrm{CO}$ (do not match the spectra). 3) Scattering of far-UV thermal emission by the early fireballs (they were relatively cold [Carlson et al., 1995]). 4) Reflection of sunlight by dust in the plume (since no continuum was observed at nearby wavelengths). 5) Finally, gaseous $\mathrm{C}$ and $\mathrm{Al}^{+}$in the plume would resonantly scatter sunlight, but modeling as done for the H-Ly $\alpha$ showed that large optical thickness effects limit each emission to only $1-2 \mathrm{kR}$.

Acknowledgments. We are very grateful to the IUE staffs at GSFC (US) and Vilspa (Spain) for their support during the SL9 observations, in particular to the observatory directors $Y$. Kondo and $W$. Wamsteker. We also thank
T. Johnson, M.M. MacLow and R. Yelle for helpful conversations. The PIs of the IUE SL9 campaign were W.M. Harris, R. Prangé, T.A. Livengood and M.A. McGrath. This research was supported by NASA grants NAGW-4006, NAG52458 and NAGW-1766 to the U. of Michigan.

\section{References}

Boslough, M.B. et al., Mass and penetration depth of ShoemakerLevy 9 fragments from time-resolved photometry, Geophys. Res. Lett., 21, 1555, 1994.

Carlson, R.W. et al, Galileo infrared observations of the Shoemaker-Levy $9 \mathrm{G}$ impact fireball: A preliminary report, Geophys. Res. Lett., 22, 1557, 1995.

Chevalier, R.A., and C.L. Sarazin, Explosions of infalling comets in Jupiter's atmosphere, Astrophys. J., 429, 863, 1994.

Clarke, J.T. et al., Hubble Space Telescope far-ultraviolet imaging of Jupiter during the impacts of Comet Shoemaker-Levy 9 , Science, 267, 1302, 1995.

Feldman, P.D., and W.G. Fastie, Fluorescence of Molecular Hydrogen Excited by Solar Extreme-Ultraviolet Radiation, Astrophys. J. Lett., 185, L101, 1973.

Gladstone, G.R., UV resonance line dayglow emissions on Earth and Jupiter, J. Geophys. Res., 93, 14,623, 1988.

Gladstone, G.R., and T.E. Skinner, Spectral Analysis of Jovian auroral emissions, Time-Variable Phenomena in the Jovian System, NASA Spec. Publ. SP-494, pp. 221, 1989.

Hammel, H.B. et al., HST Imaging of atmospheric phenomena created by the impact of comet SL9, Science, 267, 1288, 1995.

Harris, W.H. et al., Analysis of Jovian auroral H Ly-a emission (1981-1990), submitted to J. Geophys. Res., 1995.

Hill, T.W., and A.J. Dessler, Midlatitude Jovian aurora produced by the impact of Comet Shoemaker-Levy 9, Geophys. Res. Lett., 22, 1817, 1995.

Hord, C.W. et al, Direct observations of the comet ShoemakerLevy 9 fragment G impact by Galileo UVS, Geophys. Res. Lett., 22, 1565, 1995.

Ip, W.H., and R. Prangé, On possible magnetospheric dust interactions of comet Shoemaker-Levy 9 at Jupiter, Geophys. Res. Lett., 21, 1051, 1994.

Jordan, C. et al., Emission Lines of $\mathrm{H}_{2}$ in the Extreme-Ultraviolet Solar Spectrum, A strophys. J., 226, 687, 1978.

Kellogg, P.J., Plasma effects on the interaction of a comet with Jupiter, Geophys. Res. Lett., 21, 1055, 1994.

Livengood, T.A., D.F. Strobel, and H.W. Moos, Long-term study of longitudinal dependence in primary particle precipitation in the north Jovian aurora, J. Geophys. Res., 95, 10,375, 1990.

McGrath, M.A. et al., IUE observations of the Jovian dayglow emission, Geophys. Res. Lett., 16, 583, 1989.

McGregor, P., CASPIR observations from Siding Spring Observatory, Australia, IAU Colloquium 156, Baltimore, May 1995.

Nicholson, P.D. et al., Palomar observations of the R impact of comet Shoemaker-Levy 9: I. Light curves, Geophys. Res. Lett., 22, 1613, 1995.

Noll, K.S. et al., Hubble Space Telescope spectroscopic observations of Jupiter after the collision of comet P/Shoemaker-Levy 9, Science, 267, 1307, 1995.

Prangé, R. et al., Auroral signature of Comet SL9 in the Jovian Magnetosphere, Science, 267, 1317, 1995.

Waite, J.H. et al., ROSAT Observations of X-ray emissions from Jupiter during the impact of Comet Shoemaker-Levy 9, Science $268,1598,1995$.

G.E. Ballester, University of Michigan, Dept. of Atmospheric, Oceanic and Space Sciences, 2455 Hayward, Ann Arbor, MI 48109-2143, gilda@umich.edu

(received March 28, 1995; revised August 1, 1995; accepted August 7, 1995.) 